\title{
O retorno do paciente renal crônico às atividades produtivas após o transplante renal
}

\section{Patient return chronic kidney to production activities after kidney transplantation}

\author{
Nathália Cristina Silva Pereira ${ }^{1}$, Jordana Santos Cardoso Gléria ${ }^{2}$
}

http://dx.doi.org/10.11606/issn.2238-6149.v28i2p221-229

Pereira NCS, Cardoso JS. O retorno do paciente renal crônico às atividades produtivas após o transplante renal. Rev Ter Ocup Univ São Paulo. 2017 maio-ago.;28(2):221-9.

RESUMO: Introdução: O transplante renal possibilita o resgate do bem estar físico e da inserção no contexto social da pessoa com Doença Renal Crônica, em que o retorno à vida profissional e as outras atividades cotidianas se dão de forma gradativa. Objetivo: Avaliar o retorno às atividades produtivas após o transplante renal em pacientes acompanhados no Centro de Prevenção de Doenças Renais do Serviço de Nefrologia do Hospital Universitário da Universidade Federal do Maranhão. Métodos: Estudo transversal de caráter descritivo, com dados secundários oriundos de prontuários, a partir da Canadian Occupational Performance Measure (COPM) e amostra de 127 prontuários. Resultados: Predominância de pacientes do sexo masculino $(63,78 \%)$, média de idade de 41,5 anos, escolaridade com predominância de ensino fundamental incompleto $(22,05 \%)$, aposentados $(30,71 \%)$, predominância de tempo decorrido de transplante de 5 a 10 anos (47,24\%), 26,77\% permaneceram na mesma profissão após o transplante, $52,76 \%$ possuem trabalho remunerado e $11,02 \%$ não referiram retorno às atividades produtivas. A faixa etária de 35 a 55 anos e aqueles com nível de escolaridade fundamental incompleto apresentaram maior retorno às atividades produtivas. Conclusão: A amostra estudada apresentou resultados satisfatórios no que tange ao retorno às atividades produtivas, mas algumas variáveis merecem maior atenção a exemplo da escolaridade, sexo e idade quando relacionados ao retorno às atividades produtivas.

DESCRITORES: Transplante de rim; Desempenho profissional; Reabilitação vocacional.
Pereira NCS, Cardoso JS. Patient return chronic kidney to production activities after kidney transplantation. Rev Ter Ocup Univ São Paulo. 2017 May-Aug.;28(2):221-9.

ABSTRACT: Introduction: Kidney transplantation enables the rescue of physical well-being and inclusion in the social context of the person with chronic kidney disease, in which the return to work and other daily activities take place gradually. Objective: To evaluate the return to productive activities after renal transplantation in patients followed by the Center for Prevention of Kidney Disease from the Nephrology Service of the University Hospital of Federal University of Maranhão. Methods: Cross-sectional study with descriptive character, using secondary data from medical records from the Canadian Occupational Performance Measure (COPM) and samples of 127 records. Results: Predominantly male patients (63.78\%), average age of 41.5 years, schooling with a predominance of incomplete primary education $(22.05 \%)$, retired $(30.71 \%)$, predominance of elapsed time of transplantation is 5 to 10 years $(47.24 \%), 26.77 \%$ remained in the same profession after transplantation, $52.76 \%$ have paid employment and $11.02 \%$ did not report back to productive activities. The 35-55 years age group and those with incomplete primary education had higher return to productive activities. Conclusion: The study showed satisfactory results regarding the return to productive activities, but some variables like education, gender and age are important when related to the return to productive activities.

KEYWORDS: Kidney transplantation; Work performance; Rehabilitation, vocational.

Artigo apresentado como trabalho de conclusão do Programa de Residência Integrada Multiprofissional em Saúde (RIMS) do Hospital Universitário da Universidade Federal do Maranhão (HUUFMA), Turma 2013-2015. Apresentando no XI Congresso Norte e Nordeste de Terapia Ocupacional-Centenário da Prática Clínica da Terapia Ocupacional: Perspectivas Contemporâneas, Fortaleza-CE, $24-27$ out. 2016. 1. Universidade do Estado do Pará - UEPA. E-mail: nathaliacristinasp@gmail.com

2. Secretaria Municipal de Saúde, São Luís, MA. E-mail: cardoso_jordana@hotmail.com

Responsável pela correspondência: Nathália Cristina Silva Pereira. Travessa Perebebuí, n ${ }^{\circ} 2623$. Bairro Marco, Campus II - CCBS. CEP: 66087-670. E-mail: nathaliacristinasp@gmail.com 


\section{INTRODUÇÃO}

\section{Doença Renal Crônica (DRC) é considerada \\ um problema de saúde pública mundial e no} foi de 100.397 pacientes em tratamento dialítico, ocasionando em aumento dos custos de tratamento desta doença ${ }^{1}$.

De acordo com a Sociedade Brasileira de Nefrologia ${ }^{1}$, até julho de 2013 a estimativa foi de 100.397 pacientes em tratamento dialítico e conforme o Registro Brasileiro de Transplantes ${ }^{2}$, o ano de 2014 apresentou um total de 5.628 transplantes de rins no Brasil e cerca de 20 casos no Maranhão (MA).

O paciente com DRC pode vivenciar uma repentina mudança no seu cotidiano e o modo de enfrentar esta situação se torna particular para cada indivíduo, em especial para aquele que depende de tratamento dialítico ${ }^{3}$.

Além de provocar isolamento social, alterações da imagem corporal e o sentimento ambíguo entre o medo de viver e o de morrer, as repercussões da doença também acarretam ruptura na rotina diária da pessoa com DRC e entre elas podem ser citadas: a perda do emprego, a dependência financeira, as dificuldades de locomoção, a diminuição da atividade física, a necessidade de adaptação e a perda da autonomia ${ }^{4}$.

Com o transplante renal (Tx-renal) é possível resgatar o bem-estar físico e a capacidade cognitiva do paciente, além de mantê-lo inserido no contexto social, mas necessita para tal do comprometimento do paciente em seu autocuidado ${ }^{5}$. Neste sentido, o tx-renal é uma forma de terapia renal substitutiva (TRS) que prediz uma melhora na qualidade de vida, reduzindo o estresse, facilitando a vida social, o retorno a vida diária e melhora no apoio social ${ }^{6}$.

No entanto, após o processo cirúrgico do Tx-renal, percebe-se uma série de dificuldades relacionadas à reorganização do cotidiano e da vida social, sendo estas influenciadas pelas mudanças ocasionadas pela rotina hospitalar, desgaste físico e emocional consequente ao processo anterior de adoecimento.

Decorrido algum período da realização do Tx-renal e dado o início ao retorno gradativo às atividades cotidianas, o paciente é exposto as novas preocupações como as dificuldades relacionadas ao retorno às atividades produtivas, convívio com a família e outras responsabilidades, como adesão à rotina de consultas e tratamento medicamentoso $0^{6}$.

Logo, a reintegração ao trabalho é um importante parâmetro quando se analisa o impacto positivo do transplante na qualidade de vida dos receptores?
As atividades profissionais e produtivas aqui mencionadas fazem parte do contexto de desempenho ocupacional descrita pela American Occupational Therapy Association $^{8}$ classificadas como:

\footnotetext{
Administração da casa (cuidado com a roupa, limpeza da casa, preparação da comida, fazer compras, administração do dinheiro, manutenção do domicílio, procedimento de segurança), cuidar dos outros, atividades educativas e atividades vocacionais (exploração vocacional, aquisição de um trabalho, planejamento da aposentadoria, participação voluntária).
}

Assim, a cada mês decorrido do transplante renal, diminuem as restrições e os cuidados pós-cirúrgicos, possibilitando um convívio social pleno e saudável. Logo, o retorno à vida profissional e as outras atividades cotidianas se dão de forma gradativa, mas de maneira geral todos estão aptos para o retorno produtivo".

Desta forma, este estudo tem como objetivo avaliar o retorno às atividades produtivas após o transplante renal em pacientes acompanhados no Centro de Prevenção de Doenças Renais (CPDR) do Serviço de Nefrologia do Hospital Universitário da Universidade Federal do MA (HUUFMA) considerando o perfil sociodemográfico, o desempenho ocupacional, o tipo de atividade produtiva e o período decorrido após o Tx-renal.

\section{PROCEDIMENTOS METODOLÓGICOS}

Trata-se de um estudo descritivo de caráter transversal, realizado a partir de dados secundários oriundos do prontuário, a partir do instrumento de avaliação Canadian Occupational Performance Measure - COPM, aplicadas com pacientes do ambulatório do pós-transplante renal. Este instrumento foi validado no Brasil e traduzido para o português ${ }^{10}$. No local de estudo é utilizada a versão deste instrumento modificada, recebendo a nomenclatura Medida Canadense de Desempenho Ocupacional Modificada (COPM Modificada).

A COPM é utilizada para identificar dificuldades nas áreas no desempenho ocupacional, oferecer uma quantificação das prioridades de desempenho ocupacional do cliente, avaliar o desempenho e a satisfação relacionados às áreas problemas e medir as mudanças na percepção do cliente sobre seu desempenho ocupacional ao longo do programa de intervenção de terapia ocupacional ${ }^{10}$.

As atividades cotidianas enquadram-se em qualquer uma das áreas de desempenho (autocuidado, produtividade e lazer), mas para fins deste estudo foi considerada apenas a 
área de produtividade. A COPM possui classificações para a atividade produtiva, entre as quais: trabalho remunerado, trabalho não remunerado, tarefas domésticas, escola e brincar $^{11}$.

A amostra consiste em 150 avaliações contidas em prontuários de pacientes pós-transplantados, com idade entre 18 e 65 anos. Para o critério de idade foram utilizadas a idade mínima de 18 anos considerando a maior idade penal e as idades de 65 anos para homens e 60 anos para mulheres conforme o Art. 48, da Lei $\mathrm{N}^{\mathrm{o}} 8.213$, de 24 de julho de $1991^{12}$, que estabelece a idade mínima para a aposentadoria.

Os prontuários elencados são de pacientes que estiveram em acompanhamento pela Terapia Ocupacional no ambulatório de transplante renal por no mínimo seis meses, entre os anos de 2011 a 2013. Considerando que os primeiros seis meses após a cirurgia houve uma rotina de acompanhamento clínico mais intensa, pelo risco mais alto de rejeição neste período, visitas constantes e necessárias ao hospital e ajustamento do paciente à medicação e seus efeitos colaterais ${ }^{6}$.

Foram excluídos da pesquisa os prontuários que apresentavam ausência das informações necessárias para este estudo $(n=9)$, prontuários de pacientes que retornaram para hemodiálise (HD) $(\mathrm{n}=14)$, obtendo-se com isso uma amostra final de 127 prontuários.

Para fins de análise foram consideradas as seguintes variáveis: idade, sexo, estado civil, escolaridade, lugar de domicílio, benefícios previdenciários, tempo de Tx-renal, tipos de atividades produtivas e condições produtivas antes e após $\mathrm{O}$ Tx-renal. A análise descritiva dos dados foi realizada a partir de frequências e médias com o Software Stata 11.0.

As informações sobre a condição produtiva antes e após o transplante renal relatadas na parte descritiva da COPM Modificada foram listadas e categorizadas, permitindo análise descritiva.

Este estudo foi submetido ao Comitê de Ética e Pesquisa do HUUFMA sob aprovação de Parecer Consubstanciado $\mathrm{n}^{\circ}$ 932.832, em 8 de janeiro de 2015 e cumpriu os requisitos exigidos pela Resolução do Conselho Nacional de Saúde no 466/2012. No que se refere à confidencialidade dos dados do paciente, estes foram resguardados e preservados, a fim de respeitar as exigências éticas relacionadas à pesquisas com seres humanos.

\section{RESULTADOS}

Na Tabela 1 são demonstrados os dados referentes aos aspectos sociodemográficos do universo estudado.
Conforme mostra esta tabela, os pacientes tinham média de idade entre $41,5 \pm 11,5$ anos, variando de 18 a 65 anos. A maioria dos pacientes pertence à faixa etária entre os $36 \mathrm{e}$ 55 anos (49,61\%). No que se refere ao sexo, o grupo consistiu de $81(63,78 \%)$ pacientes do sexo masculino e $46(36,22 \%)$ pacientes do sexo feminino. Com referência ao lugar de domicílio, 74 (58,27\%) pacientes residem na capital.

Um dado significativo diz respeito à escolaridade, na qual $28(22,05 \%)$ pacientes apresentaram ensino fundamental incompleto e $27(21,26 \%)$ ensino médio completo. No que tange ao estado civil, a maioria são casados, totalizando $73(57,48 \%)$ e $33(25,98 \%)$ solteiros, seguido de união estável, divorciado e viúvo.

Em relação aos benefícios previdenciários, 39 $(30,71 \%)$ são aposentados, seguido do auxílio-doença $(24,11 \%)$ e beneficiado pelo Benefício de Prestação Continuada $(8,66 \%)$ (Tabela 1$)$. Quanto à variável tempo decorrido do Tx-renal, 60 (47,24\%) pacientes tem entre 5 e 10 anos de transplante, seguido de $42(33,07 \%)$ pacientes acima de 10 anos e $25(19,69 \%)$ pacientes entre 1 e 5 anos de transplante.

Na Tabela 2 são demonstrados também os dados referentes aos tipos de atividades produtivas. Observouse que $67(52,76 \%)$ dos pacientes possuem trabalho remunerado, 18 (14,17\%) realizam duas ou mais atividades produtivas e $16(12,60 \%)$ realizam apenas as tarefas domésticas. Faz-se importante destacar que apenas $14(11,02 \%)$ das avaliações não apresentaram dados referentes ao retorno das atividades produtivas.

Ainda com relação à variável tipo de atividades produtivas, entre aqueles que citaram que o retorno às atividades correspondeu a duas ou mais atividades, entre as mais citadas tem-se: trabalho não remunerado e tarefas domésticas; trabalho remunerado e faculdade; trabalho remunerado e tarefas domésticas; trabalho remunerado e trabalho não remunerado; faculdade e tarefas domésticas.

A variável condição produtiva antes e após o $T x$-renal revela que $34(26,77 \%)$ pacientes permanecem com a mesma profissão e/ou referiu novos projetos e 31 $(24,41 \%)$ pacientes apresentaram mudança profissional (Tabela 2).

A Tabela 3 apresenta dados referentes à relação entre sexo, idade, escolaridade e atividades produtivas após Tx-renal. Conforme a tabela é possível observar que o sexo masculino apresentou um alto percentual de retorno quanto comparado ao sexo feminino, principalmente no que diz respeito ao retorno ao trabalho remunerado totalizando $45(67,16 \%)$. Faz-se importante destacar que dos $14(11,02 \%)$ pacientes que não retornaram as atividades produtivas, todos eram do sexo masculino. 
Pereira NCS, Cardoso JS. O retorno do paciente renal crônico às atividades produtivas. Rev Ter Ocup Univ São Paulo. 2017 maio/ago.;28(2):221-9.

Tabela 1 - Características sociodemográficas de pacientes submetidos ao transplante renal no HUUFMA, São Luís, MA, 2015

\begin{tabular}{|c|c|c|}
\hline VARIÁVEIS & n & $\%$ \\
\hline \multicolumn{3}{|l|}{ Idade (em anos) } \\
\hline$\leq 35$ & 46 & 36,22 \\
\hline$>35-\leq 55$ & 63 & 49,61 \\
\hline$>55-\leq 65$ & 18 & 14,17 \\
\hline \multicolumn{3}{|l|}{ Sexo } \\
\hline Masculino & 81 & 63,78 \\
\hline Feminino & 46 & 36,22 \\
\hline \multicolumn{3}{|l|}{ Lugar de domicílio } \\
\hline Sem identificação & 1 & 0,79 \\
\hline São Luís & 74 & 58,27 \\
\hline Interior & 48 & 37,80 \\
\hline Outros & 4 & 3,15 \\
\hline \multicolumn{3}{|l|}{ Escolaridade } \\
\hline Sem identificação & 15 & 11,81 \\
\hline Analfabeto & 2 & 1,57 \\
\hline Analfabeto funcional & 5 & 3,94 \\
\hline Ens. fundamental incompleto & 28 & 22,05 \\
\hline Ens. fundamental completo & 10 & 7,87 \\
\hline Ens. médio incompleto & 9 & 7,09 \\
\hline Ens. médio completo & 27 & 21,26 \\
\hline Ens. superior incompleto & 14 & 11,02 \\
\hline Ens. superior completo & 17 & 13,39 \\
\hline \multicolumn{3}{|l|}{ Estado civil } \\
\hline Sem identificação & 3 & 2,36 \\
\hline Solteiro (a) & 33 & 25,98 \\
\hline União estável & 12 & 9,45 \\
\hline Casado (a) & 73 & 57,48 \\
\hline Divorciado (a) & 4 & 3,15 \\
\hline Viúvo (a) & 2 & 1,57 \\
\hline \multicolumn{3}{|l|}{ Benefícios previdenciários } \\
\hline Sem identificação & 46 & 36,22 \\
\hline $\mathrm{BPC}$ & 11 & 8,66 \\
\hline Auxílio-doença & 31 & 24,41 \\
\hline Aposentadoria & 39 & 30,71 \\
\hline \multicolumn{3}{|l|}{ Tempo de tx renal (em anos) } \\
\hline$\geq 1-<5$ & 25 & 19,69 \\
\hline$\geq 5-<10$ & 60 & 47,24 \\
\hline$\geq 10$ & 42 & 33,07 \\
\hline Total & 127 & 100 \\
\hline
\end{tabular}

Tabela 2 - Tipo de atividades produtivas e condição produtiva de pacientes submetidos ao transplante renal no HUUFMA, São Luís, MA, 2015

\begin{tabular}{lcc}
\hline VARIÁVEIS & $\mathbf{n}$ & $\mathbf{\%}$ \\
\hline Tipo de atividades produtivas & & \\
Não retornou as atividades produtivas & 14 & 11,02 \\
Trabalho remunerado & 67 & 52,76 \\
Trabalho não remunerado & 3 & 2,36 \\
Tarefas domésticas & 16 & 12,60 \\
Escola/Faculdade & 9 & 7,09 \\
Duas ou + atividades & 18 & 14,17 \\
Condição produtiva antes e após o tx renal & \\
Sem projetos & 28 & 22,05 \\
Mesma profissão & 34 & 26,77 \\
Mudança profissional & 31 & 24,41 \\
Novos projetos de vida & 34 & 26,77 \\
Total & $\mathbf{1 2 7}$ & $\mathbf{1 0 0}$ \\
\hline
\end{tabular}

Quanto ao sexo feminino o mesmo apresentou percentual de retorno no que tange ao trabalho remunerado (22/32,84\%), duas ou mais atividades $(12 / 66,67 \%)$ e nas tarefas domésticas $(9 / 56,25 \%)$ (Tabela 3$)$.

Conforme a Tabela 3, ainda é possível observar a relação entre faixa etária e retorno às atividades produtivas, de um modo geral o retorno às atividades está mais presente na faixa etária entre 35 e 55 anos totalizando 63 (49,61\%).

Esta mesma faixa etária apresentou maior percentual de retorno às atividades seguintes: trabalho não remunerado $2(66,67 \%)$ pacientes, duas ou mais atividades $10(55,56 \%)$ pacientes, trabalho remunerado 35 (52,24\%) pacientes e tarefas domésticas 7 (43,75\%) pacientes. A faixa etária de $\leq 35$ anos apresenta um retorno mais significativo com relação à escola ou faculdade totalizando $7(77,78 \%)$ pacientes.

Ainda com relação à Tabela 3 , o nível de escolaridade que apresentou maior percentual total em exercício das atividades produtivas foi o ensino fundamental incompleto totalizando $28(22,05 \%)$ pacientes, seguido do ensino médio completo $27(21,26 \%)$. No que se refere à variável retorno ao trabalho remunerado, pacientes que possuem ensino fundamental incompleto 16 (23,88\%) e ensino médio completo $14(20,90 \%)$ apresentaram maior percentual de retorno. Pacientes com ensino médio completo $2(66,67 \%)$ apresentaram maior percentual de retorno ao trabalho não remunerado. Já no que diz respeito ao retorno às tarefas domésticas, pacientes com ensino fundamental incompleto $6(37,50 \%)$ são aquelas que mais retornaram para esta atividade. 
Pereira NCS, Cardoso JS. O retorno do paciente renal crônico às atividades produtivas. Rev Ter Ocup Univ São Paulo. 2017 maio/ago.;28(2):221-9.

Tabela 3 - Relação das variáveis sexo, idade e escolaridade relacionadas aos tipos de atividades produtivas de pacientes submetidos ao transplante renal no HUUFMA, São Luís, MA, 2015

\begin{tabular}{|c|c|c|c|c|c|c|c|}
\hline \multirow{3}{*}{ VARIÁVEIS } & \multicolumn{7}{|c|}{ ATIVIDADES PRODUTIVAS } \\
\hline & $\begin{array}{c}\text { Não retornou } \\
\text { as atividades } \\
\text { produtivas }\end{array}$ & $\begin{array}{c}\text { Trabalho } \\
\text { remunerado }\end{array}$ & $\begin{array}{l}\text { Trabalho não } \\
\text { remunerado }\end{array}$ & $\begin{array}{c}\text { Tarefas } \\
\text { domésticas }\end{array}$ & $\begin{array}{c}\text { Escola/ } \\
\text { Faculdade }\end{array}$ & $\begin{array}{l}\text { Duas ou }+ \\
\text { atividades }\end{array}$ & Total \\
\hline & n (\%) & n (\%) & n (\%) & n (\%) & n (\%) & n (\%) & n (\%) \\
\hline Sexo & 14 & 67 & 3 & 16 & 9 & 18 & 127 \\
\hline Masculino & $14(11,02)$ & $45(67,16)$ & $2(66,67)$ & $7(43,75)$ & $7(77,78)$ & $6(33,33)$ & $81(63,78)$ \\
\hline Feminino & $0(0)$ & $22(32,84)$ & $1(33,33)$ & $9(56,25)$ & $2(22,22)$ & $12(66,67)$ & $46(36,22)$ \\
\hline \multicolumn{8}{|l|}{ Idade } \\
\hline$\leq 35$ & $3(21,43)$ & $25(37,31)$ & $0(0)$ & $5(31,25)$ & $7(77,78)$ & $6(33,33)$ & $46(36,22)$ \\
\hline$>35-\leq 55$ & $7(50)$ & $35(52,24)$ & $2(66,67)$ & $7(43,75)$ & $2(22,22)$ & $10(55,56)$ & $63(49,61)$ \\
\hline$>55-\leq 65$ & $4(28,57)$ & $7(10,45)$ & $1(33,33)$ & $4(25)$ & $0(0)$ & $2(11,11)$ & $18(14,17)$ \\
\hline \multicolumn{8}{|l|}{ Escolaridade } \\
\hline Sem identificação & $0(0)$ & $13(19,40)$ & $0(0)$ & $0(0)$ & $0(0)$ & $2(11,11)$ & $15(11,81)$ \\
\hline Analfabeto & $1(7,14)$ & $0(0)$ & $0(0)$ & $0(0)$ & $0(0)$ & $1(5,56)$ & $2(1,57)$ \\
\hline Analfabeto funcional & $1(7,14)$ & $1(1,49)$ & $1(33,33)$ & $2(12,50)$ & $0(0)$ & $0(0)$ & $5(3,94)$ \\
\hline $\begin{array}{l}\text { Ens. fundamental } \\
\text { incompleto }\end{array}$ & $4(28,57)$ & $16(23,88)$ & $0(0)$ & $6(37,50)$ & $1(11,11)$ & $1(5,56)$ & $28(22,05)$ \\
\hline Ens. fundamental completo & $2(14,29)$ & $3(4,48)$ & $0(0)$ & $3(18,75)$ & $0(0)$ & $2(11,11)$ & $10(7,87)$ \\
\hline Ens. médio incompleto & $1(7,14)$ & $3(4,48)$ & $0(0)$ & $2(12,50)$ & $1(11,11)$ & $2(11,11)$ & $9(7,09)$ \\
\hline Ens. médio completo & $3(21,43)$ & $14(20,90)$ & $2(66,67)$ & $1(6,25)$ & $2(22,22)$ & $5(27,78)$ & $27(21,26)$ \\
\hline Ens. superior incompleto & $0(0)$ & $5(7,46)$ & $0(0)$ & $0(0)$ & $5(55,56)$ & $4(22,22)$ & $14(11,02)$ \\
\hline Ens. superior completo & $2(14,29)$ & $12(17,91)$ & $0(0)$ & $2(12,50)$ & $0(0)$ & $1(5,56)$ & $17(13,39)$ \\
\hline Total & $14(100 \%)$ & $67(100 \%)$ & $3(100 \%)$ & $16(100)$ & $9(100 \%)$ & $18(100 \%)$ & $127(100 \%)$ \\
\hline
\end{tabular}

Do total de pacientes, $5(55,56 \%)$ estão com o ensino superior em andamento. A escolaridade de ensino médio completo apresentou percentual de $5(27,78 \%)$ para o exercício de duas ou mais atividades, seguido de 4 $(22,22 \%)$ de ensino superior incompleto.

\section{DISCUSSÃO}

$\mathrm{O}$ retorno às atividades produtivas após o transplante renal pode ocorrer em ritmo lento, com avanços e recuos, além de apresentar obstáculos como as complicações clínicas que nem sempre podem ser prevenidas e controladas ${ }^{13}$. No entanto, a restauração da capacidade funcional e a diminuição das restrições da diálise são possibilidades oferecidas pelo transplante renal que quando bem sucedido, possibilita aos sujeitos a redução de tal restrição e permite maior independência, recuperando desta forma as capacidades existentes antes da $\mathrm{DRC}^{14}$.

Neste estudo é possível demonstrar um retorno significativo às atividades produtivas, principalmente ao trabalho remunerado conforme mostra a Tabela 2. Este dado permite inferir que houve um aumento da capacidade funcional do paciente e melhora da independência nas atividades cotidianas após o tx renal, aspecto também demonstrado na Tabela 1 no que se refere à manutenção da mesma atividade profissional, ingresso em nova atividade profissional ou na projeção de projetos de vida.

Godoy et al. ${ }^{15}$ afirmam que a melhora da capacidade funcional após o transplante oferece ganhos no aspecto físico e mental. Este autor também evidenciou que o retorno a qualquer atividade produtiva proporciona também repercussões tanto do ponto de vista social como individual, aumentando a autoestima e as relações sociais.

Um estudo ${ }^{16}$ realizado na mesma instituição da presente pesquisa, corrobora os dados encontrados no que se refere ao retorno as atividades produtivas, destacando-se que $77,3 \%$ pacientes transplantados renais realizam alguma ocupação.

Como mostra Tabela 1 foi possível perceber que o maior percentual de pacientes que retornaram ao trabalho está entre aqueles pacientes que possuem tempo 
decorrido do Tx renal acima de 5 anos e entre aqueles que mantiveram a mesma atividade profissional (Tabela 3). De acordo com Costa $^{17}$ as pessoas que se encontravam em atividade produtiva antes do transplante regressaram mais rapidamente ao trabalho remunerado.

A condição produtiva de encontrar-se na mesma profissão, os novos projetos e as atividades produtivas de trabalho remunerado revelados neste estudo, corroboram outro estudo no qual é demonstrada que a origem da renda tem sido o trabalho remunerado e o tipo de atividade laboral após o transplante é o trabalho autônomo ${ }^{18}$.

No estudo supracitado são mencionadas como tipo de atividade laboral realizadas após o transplante renal as seguintes ocupações: trabalho autônomo, comerciário, professor, lavrador, motorista, auxiliar administrativo, auxiliar de escritório.

No presente estudo, o retorno às atividades produtivas foi mais encontrado em pacientes do sexo masculino e este dado corrobora outros estudos presentes na literatura ${ }^{19,20,21}$. Neste sentido, é importante destacar um estudo descritivo composto por 265 transplantados renais, a maioria do sexo masculino $(62,1 \%)^{22}$ que apresentou dados compatíveis com esta pesquisa.

A predominância de retorno à atividade produtiva em pacientes do sexo masculino pode estar relacionado ao papel desempenhado pelo homem enquanto provedor da família, o que é possível inferir a partir do alto percentual de pacientes casados, do sexo masculino e do tipo de atividade à qual os pacientes do sexo masculino apresentaram maior frequência (trabalho remunerado e escola/faculdade).

No que se refere à faixa etária, a média de idade encontrada em alguns estudos ${ }^{21,22}$ é de 40 anos, o que corrobora com os dados desta pesquisa, a qual demonstrou este resultado na faixa etária entre $>35$ e $\leq 55$ dos pacientes estudados. Este dado permite inferir que muitos dos pacientes que retornam às atividades se encontram em idade produtiva ${ }^{23}$ e esta variável também é componente facilitador para o retorno ao trabalho.

Faz-se importante destacar que os pacientes mais jovens com idade entre 18 e 35 anos apresentaram um percentual de retorno às atividades abaixo da faixa etária acima citada, principalmente no que se refere às atividades escolares ou ingresso em ensino superior, já que esta faixa etária se encontra no período em que poderia haver investimentos para ingressar nestas atividades, causa que deve ser melhor investigada em novos estudos.

$\mathrm{O}$ fato de pacientes mais idosos (18 pacientes) retornarem com menor frequência ao trabalho não é contrário a outros estudos, visto que estes buscam a aposentadoria mais precocemente e sua habilidade em encontrar emprego decresce com a idade ${ }^{7}$, assim como absorção do mercado de trabalho para esta faixa etária.

A predominância do lugar de domicílio na capital corresponde à $74(58,27 \%)$ pacientes e também corrobora outro estudo presente na literatura ${ }^{18}$. O local de residência também pode influenciar no retorno ao trabalho, já que em capitais a oferta de trabalho remunerado e os meios de ingresso e de continuidade da carreira educacional são mais diversificados, o que possibilita mais opções por meios de sobrevivência como o trabalho formal ou o trabalho informal.

Além disso, a permanência na capital facilita o acesso aos serviços de saúde, ao saneamento básico e às melhores condições de vida, o que implica diretamente na qualidade de vida do paciente e na manutenção de hábitos de vida saudáveis, condição essencial para o sucesso do transplante e por conseguinte, para o retorno ao trabalho ${ }^{24}$.

Quanto a variável escolaridade (Tabela 1), o estudo demonstrou predominância de pacientes com o ensino fundamental incompleto e ensino médio completo, dado que correspondente à outra pesquisa também realizada num hospital universitário na qual foi apresentado o mesmo resultado ${ }^{22}$ e reflete a realidade brasileira, na qual grande parcela da população tem poucos anos de estudo ${ }^{25}$.

O achado relacionado ao ensino médio é considerado um dado positivo, já que este nível de escolaridade demonstra também um perfil de empregabilidade ou de ingresso em instituições educacionais, pois muitas instituições exigem o ensino médio completo como pré-requisito para contratação, assim como esta escolaridade é um pré-requisito para o ingresso ao ensino técnico profissionalizante, ao ensino superior e em alguns cursos de capacitação.

Alguns estudos ${ }^{18,21}$ indicam que o retorno ao trabalho é mais evidente entre indivíduos com maior grau de escolaridade. Na Tabela 3 é possível identificar esta relação, ao se comparar a frequência de ingresso em atividades produtivas de pacientes com ensino médio completo e aqueles sem a conclusão do ensino médio, podendo-se inferir que aqueles que possuem o ensino médio completo obtiveram maior êxito quanto à inclusão em atividades produtivas remuneradas.

Somado a isso, ao se observar o perfil dos pacientes com o maior nível de escolaridade (superior incompleto e superior completo) é possível constatar que não apresentaram o não retorno para o ensino superior e apenas $2(14,29 \%)$ pacientes não retornaram ao trabalho. É possível perceber também que o trabalho não remunerado não consta como atividade exercida por aqueles que têm nível superior.

O estudo revelou um alto percentual de pacientes aposentados. Esta variável indica que alguns pacientes 
são submetidos à aposentadoria por invalidez ainda durante a terapia dialítica ou a opção por aposentadoria é apresentada com mais precocidade em decorrência do tipo de trabalho exercido antes do transplante renal que consistia em tarefas que exigiam maior esforço físico ${ }^{18}$.

Alguns estudos indicam que a condição de beneficiário também dificulta $\mathrm{o}$ retorno ou o acesso ao mercado formal de trabalho ${ }^{25,26}$, o que foi possível constatar em alguns relatos disponíveis na COPM, principalmente com relação aos pacientes que trabalhavam em empresas de grande porte como multinacionais, mas que a atividade laboral requeria um grande esforço físico ou exposição à situações de risco como espaços confinados, grandes alturas ou exposição à substâncias químicas.

Alguns estudos ${ }^{25,26}$ indicam que os vínculos informais acomodam melhor os transplantados renais, podendo este escolher a atividade que melhor se adapta as suas capacidades, ao seu estado geral, horários e períodos mais flexíveis. Os dados desta pesquisa corroboram os estudos citados acima, já que a partir da COPM, foi possível listar as atividades entre as quais os pacientes optaram para uma mudança profissional após o transplante renal.

Neste estudo é possível perceber um dado que não está de acordo com outros estudos encontrados na literatura, no qual apenas $14(11,02 \%)$ pacientes não retornaram as atividades produtivas. A literatura apresenta um percentual muito maior de não retorno ao trabalho, no qual é possível perceber uma estimativa de $50 \%$ a $83 \%$ de transplantados renais que nunca retornaram ao trabalho ${ }^{21}$.

Este resultado pode estar relacionado ao trabalho multiprofissional disponível no ambulatório de seguimento após o transplante renal, principalmente aquele em que são desenvolvidos trabalhos de sensibilização e resgate da motivação do paciente quanto ao ingresso, manutenção ou retorno as atividades produtivas e no qual são reforçadas as orientações relacionadas ao autocuidado do paciente transplantado renal ${ }^{27}$.

Já que apesar de desejarem regressar ao trabalho, os pacientes apresentam muitas dificuldades e necessitam de apoio no período de transição entre um período de

\section{REFERÊNCIAS}

1. Sociedade Brasileira de Nefrologia. Censo de diálise SBN 2013. São Paulo; 2013.

2. Associação Brasileira de Transplantes de Órgãos (ABTO). RBT - Registro Brasileiro de Transplantes. Dados restrições prévias ao transplante e o regresso a um estilo de vida semelhante ao que tinham antes dos problemas de saúde surgirem ${ }^{24}$.

\section{CONCLUSÃO}

Este estudo permitiu conhecer a realidade de um hospital universitário em relação ao retorno de seus pacientes após transplante renal, apresentando resultados positivos, com alto índice de retorno as atividades produtivas.

Cabe aqui destacar que o referencial teórico que aborda atividade produtiva designa que a mesma não se relaciona apenas ao trabalho formal, mas ao trabalho informal, o ingresso na vida acadêmica e/ou escolar e na inserção nas tarefas domésticas e de cuidador, o que reflete que o retorno produtivo pode se dar de forma mais ampla.

Percebe-se com isso que o retorno às atividades produtivas é uma referência no que diz respeito à melhora da capacidade funcional e do desempenho ocupacional e um forte indicador de sucesso do transplante renal, mas que para estes sejam alcançados são necessárias inúmeras medidas terapêuticas, políticas e institucionais a serem tomadas.

Entre estas medidas destaca-se a contribuição do acompanhamento multiprofissional e contínuo no pós Tx-renal não só focando nas condições clínicas, mas também nas condições sociais, entendidas como primordiais para o encorajamento do retorno as atividades produtivas $\mathrm{e}$ consequente melhora na qualidade de vida, visto que durante algum tempo um grande número de pacientes, mantiveramse afastados das atividades cotidianas e laborais.

Faz-se necessário também a interlocução dos serviços de saúde com as instâncias responsáveis pela idealização e execução de outras políticas públicas que não da saúde, a fim de possibilitar o reingresso ao trabalho.

Dessa forma, este estudo aponta alguns dados que corroboram a proposta acima citada, mas se faz necessário mais estudos, principalmente estudos longitudinais, a fim de identificar a partir de um recorte temporal, as variáveis que interferem no retorno ao trabalho após o Tx-renal, principalmente com a população aqui estudada. 
3. Barbosa GS, Valadares GV. Hemodiálise: estilo de vida e a adaptação do paciente. Acta Paul Enferm. 2009;22(Esp. Nefrol.):524-7. doi: http://dx.doi.org/10.1590/S010321002009000800014.

4. Machado LRC, Car MR. A dialética da vida cotidiana de doentes com insuficiência renal crônica: entre o inevitável e o casual. Rev Esc Enferm USP. 2003;37(3):27-35. doi: http://dx.doi.org/10.1590/S0080-62342003000300004.

5. Proença MC, Ribeiro A, Veronese FJV, Echer IC, Gonçalves LFS, Manfro RC. Transplante renal: orientações para pacientes e familiares. In: Congresso de Pesquisa e Desenvolvimento em Saúde do Mercosul; 11 set 2004; Hospital das Clínicas de Porto Alegre, Porto Alegre, RS; 2004. Rev HCPA. Disponível em: http://hdl.handle. net/10183/1165.

6. Ravagnani LMB, Domingos NAM, Miyazaki MCOS. Qualidade de vida e estratégias de enfrentamento em pacientes submetidos a transplante renal. Estud Psicol (Natal). 2007;12(2):177-18. doi: 10.1590/S1413294X2007000200010.

7. Parolin MB, Coelho JCU, Costa PB, Pimentel SK, Santos-Neto LE, Vayego SA. Retorno ao trabalho de pacientes adultos submetidos a transplante de fígado. Arq Gastroenterol. 2001;38(3):172-5. doi: http://dx.doi. org/10.1590/S0004-28032001000300006.

8. AOTA American Occupational Therapy Association. Occupational therapy association practice framework: domain and process. Am J Ocup Ther. 2002;48:1047-54. doi: 10.5014/ajot.56.6.609.

9. ABTO. Associação Brasileira de Transplante de Órgãos. Manual de Transplante Renal: período pós-transplante. São Paulo: Novartis; s.d. Disponível em: http://www.abto.org.br/ abtov03/Upload/file/manual_transplante_pos.pdf.

10. Law M, Baptiste S, Carswell A, Mccoll MA, Polatajko HL, Pollock N. Medida Canadense de Desempenho Ocupacional (COPM). Magalhães LC, Magalhães LV, Cardoso AA, tradutores, organizadores. Belo Horizonte: Editora Universidade Federal de Minas Gerais; 2009.

11. Mccoll MA, Law M, Baptiste S, Pollock N, Carswell A, Polatajko HL. Targeted applications of the Canadian occupational performance measure. Can J Occup Ther. 2005;72(5):298-300. doi: 10.1177/000841740507200506.

12. Brasil. Presidência da República. Subchefia para Assuntos Jurídicos. Lei No 8.213, de julho de 1991. Dispõe sobre os Planos de Benefícios da Previdência Social e dá outras providências. Diário Oficial da União, 11 abr. 1996.

13. Oliveira EA. Qualidade de vida de pacientes submetidos ao transplante de medula óssea alogênico: um estudo longitudinal [Tese]. Ribeirão Preto (SP): FFCLRP/USP; 2004.
14. Valderrábano F, Jofre R, Lopez-Gomes JM. Quality of life in end-stage renal disease patients. Am J Kidney Dis. 2001;38(3):443-64. doi: http://dx.doi.org/10.1053/ ajkd.2001.26824.

15. Godoy MR, Balbinotto NG, Barros PP, Ribeiro EP. Estimando as perdas de rendimento devido à doença renal crônica no Brasil. Saúde Debate 2007;38:68-85. doi: http:// dx.doi.org/10.1590/S1413-294X2007000200010.

16. Berrêdo VCM, Carvalho CS. Qualidade de vida de pacientes transplantados renais do Hospital Universitário Unidade Presidente Dutra (HU-UPD) [Trabalho de conclusão de curso]. São Luís (MA): Universidade Federal do Maranhão; 2003.

17. Costa SRSCM. Regresso ao trabalho do doente submetido a transplante hepático [Dissertação]. Coimbra: Universidade de Coimbra; 2009.

18. Costa JM, Nogueira LT. Associação entre trabalho, renda e qualidade de vida de receptores de transplante renal no município de Teresina, PI, Brasil. J Bras Nefrol. 2014;36(3):332-38. doi: http://dx.doi.org/10.5935/01012800.20140048 .

19. Lanza AHB, Chaves APA, Garcia RCP, Brandão JAG. Biopsychological and social profile of cronic renal patients in hemodialysis. Arq Bras Cien Saúde. 2009;33(3):141-5. Disponível em: http://files.bvs.br/upload/S/1983-2451/2008/ v33n3/a141-145.pdf.

20. Lôbo MCSG, Bello VAO. Professional rehabilitation after kidney transplantation. J Bras Nefrol, 2007;29:29-32. doi: http://dx.doi.org/10.1590/S1413-294X2007000200010.

21. Lira ALBC, Lopes MVO. Pacientes transplantados renais: análise de associação dos diagnósticos de enfermagem. Rev Gaúcha Enferm (Porto Alegre). 2010;31(1):108-14. doi: http://dx.doi.org/10.1590/S1983-14472010000100015.

22. Santos PR. Relação do sexo e da idade com nível de qualidade de vida em renais crônicos hemodialisados. Rev Assoc Med Bras. 2006;52:356-9. doi: http://dx.doi. org/10.1590/S0104-42302006000500026.

23. Instituto Brasileiro de Geografia e Estatística (IBGE). Síntese de indicadores sociais 2009. Rio de Janeiro: IBGE; 2011.

24. Fontoura FAP. A compreensão de vida de pacientes submetidos ao transplante renal: significados, vivências e qualidade de vida [Dissertação]. Campo Grande (MS): Universidade Católica Dom Bosco; 2012.

25. Pontes L, Guirardello EB, Campos CJ. Demands for attention experienced by a patient in a bone marrow transplants unit. Rev Esc Enferm USP. 2007;41:154-60. doi: http://dx.doi.org/10.1590/S008062342007000100021 . 
Pereira NCS, Cardoso JS. O retorno do paciente renal crônico às atividades produtivas. Rev Ter Ocup Univ São Paulo. 2017 maio/ago.;28(2):221-9.

26. Siqueira JPFH. Benefícios decorrentes de auxílio-doença para empregados rurais, urbanos e domésticos: anotações à legislação básica pertinente. Rev Síntese Direito Previdenciário. 2011;263:71-86. doi: Disponível em: http:// bdjur.tjdft.jus.br/xmlui/handle/123456789/8047.
27. Cardoso JS, Cavalcante MCV, De Miranda ATM. A reabilitação profissional como proposta de intervenção da terapia ocupacional. Rev Ter Ocup Univ São Paulo. 2013;24(2):162-7. doi: http://dx.doi.org/10.11606/issn.22386149.v24i2p162-167.

Recebido em: 09.11.2017

Aceito em: 31.07.2017 